\title{
Counteracting Rotor Imbalance in a Bearingless Motor System with Feedforward Control
}

\author{
Peter Kascak, Ralph Jansen, Timothy Dever, Aleksandr Nagorny, Kenneth Loparo
}

\begin{abstract}
In standard motor applications, traditional mechanical bearings represent the most economical approach to rotor suspension. However, in certain high performance applications, rotor suspension without bearing contact is either required or highly beneficial. Such applications include very high speed, extreme environment, or limited maintenance access applications. This paper extends upon a novel bearingless motor concept, in which full five-axis levitation and rotation of the rotor is achieved using two motors with opposing conical air-gaps. By leaving the motors' pole-pairs unconnected, different d-axis flux in each pole-pair is created, generating a flux imbalance which creates lateral force. Note this is approach is different than that used in previous bearingless motors, which use separate windings for levitation and rotation. This paper will examine the use of feedforward control to counteract synchronous whirl caused by rotor imbalance. Experimental results will be presented showing the performance of a prototype bearingless system, which was sized for a high speed flywheel energy storage application, with and without feedforward control.
\end{abstract}

Index Terms - Magnetic Levitation, Energy Conversion, Motors, Permanent Magnet Motors, Rotating Machines, Motor Drives, Energy Storage, Flywheels.

\section{INTRODUCTION}

$I^{\mathrm{N}}$ $\mathrm{N}$ very high performance motor applications, suspension of the rotor by non-contacting bearings is desired. Benefits include reduction or elimination of maintenance requirements, enabling higher rotor speeds, reducing losses, and enabling performance in extreme environments. One way of accomplishing non-contact suspension is by using magnetic bearings. Traditionally this involves using separate actuators, in which iron and copper parts are dedicated to only one function, either levitation or rotation. This would be limiting in applications which require a large amount of levitation force in some modes of operation, and a large amount of motor power in others. Furthermore, these actuators increase the axial length of the rotor, which lowers the bending mode frequencies, complicating control of the system. Using the

P. Kascak is with NASA Glenn Research Center, Cleveland, OH 44135 USA (e-mail: peter.e.kascak@nasa.gov).

R. Jansen is with NASA Glenn Research Center, Cleveland, OH 44135 USA (e-mail: ralph.h.jansen@nasa.gov).

T. Dever is with NASA Glenn Research Center, Cleveland, OH 44135 USA (e-mail: tdever@nasa.gov).

A. Nagorny is with ResMed Motor Technologies Inc., Chatsworth, CA 91311 USA (e-mail: AleksandrN@ResMed.com).

$\mathrm{K}$. Loparo is with the Department of Electrical Engineering and Computer Science, Case Western Reserve University, Cleveland, OH 44106 USA (email: kal4@case.edu) motor for both rotation and levitation would avoid these problems, and allow the added advantage of reducing the system part count.

Bearingless motors have been demonstrated in synchronous reluctance [1], [2], induction [3], permanent magnet [4], and switched reluctance [5] configurations. These motors have common iron structures carrying both levitation and rotation flux; however, they use different windings for levitation and rotation. So each function is still limited by the windings and power electronics, which are dedicated to a single function. Furthermore, they are only capable of providing levitation forces in a radial plane in the case of radial motors, or in the axial direction for axial motors.

This paper examines the performance of a new bearingless motor concept, which was previously described by the authors of this paper in [6]-[12]. In this concept, the motor pole-pairs are separated, causing a flux imbalance which creates a levitation force. This approach allows all of the motor iron and copper to be used for either the levitation or rotation function. Additionally, since these motors have a conical shaped air-gap, they can create axial as well as radial forces. A pair of these motors with opposing cones is used to provide full 5-axis levitation.

The contribution of this paper is examining the use of feedforward to counteract synchronous whirl exhibited by the rotor of a prototype bearingless machine. The synchronous whirl is caused by a predictable force due to rotor imbalance. Because the synchronous whirl is predictable, a counter acting feedforward signal can be added to the levitation controller to counteract this effect. The basic concept of use of a feedforward signal to counteract rotor imbalance in a magnetically levitated flywheel system is described in [14].

This paper will first briefly describe the prototype bearingless machine. Next it will examine the physics of the synchronous whirl. Then finally it will be shown how a feedforward signal can be applied, and show the experimental results of adding this signal.

\section{Prototype BeAringless Machine}

A standard motor is wound with the pole-pairs connected in series or parallel, as shown in Fig. 1. In this configuration the pole-pair flux can be increased or decreased, but pole flux cannot be controlled independently, since the poles carry the same current. Thus it is not possible to create a flux imbalance on the rotor, which means that the attractive magnetic force from each pole-pair is the same; when added 
vectorially on the rotor they create zero net force. In the case of motors with rotors suspended by mechanical bearings, this balanced rotor flux is highly desirable because it reduces vibrations due to induced net forces.

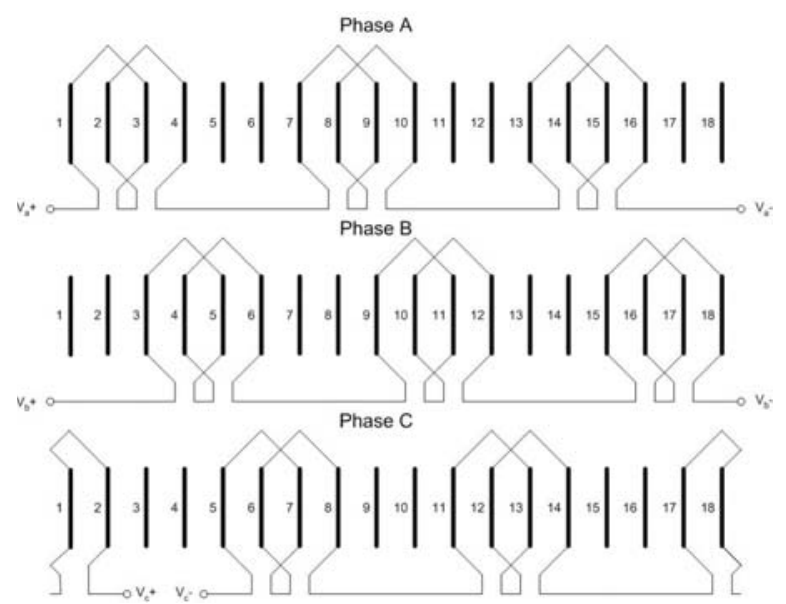

Fig. 1. Pole-Pairs Connected in Series

To create a levitating lateral force, it is necessary to generate an imbalance in the rotor. This is achieved in the prototype motors by leaving the motor pole-pairs unconnected, as shown in Fig. 2. Now the flux in each polepair can be controlled independently.

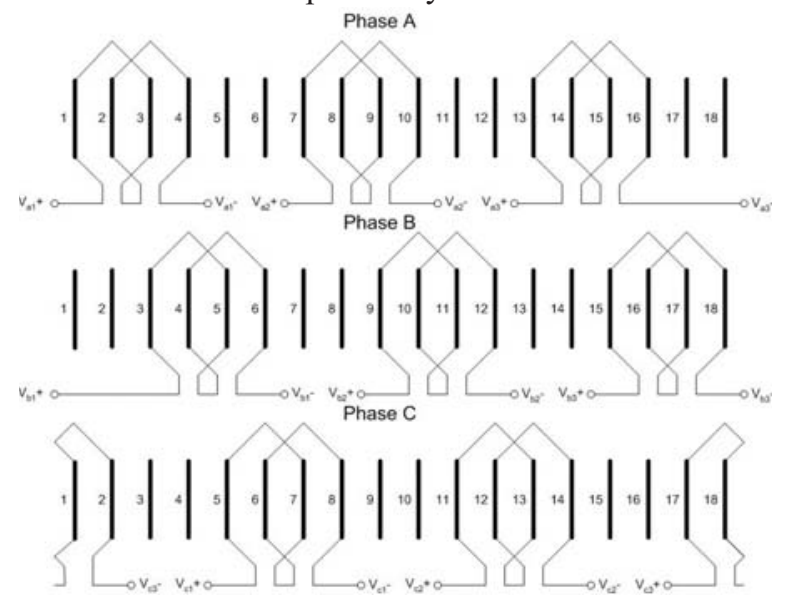

Fig. 2. Independent Pole-Pair Windings

Each pole-pair is controlled using Field Oriented Control (FOC). In FOC, the flux and torque are controlled by transforming stationary reference frame quantities (currents, flux, voltage, etc.) to the rotor reference frame. Fig. 3 shows the motor windings represented in the rotor reference frame: the d-axis is directed into the magnet, and the q-axis is directed 90 electrical degrees ahead of the d-axis. The d-axis controls the flux, and the q-axis controls the torque. By using different rotor reference frame d-axis currents in each polepair, a flux differential can be created on the rotor. Using finite element analysis, the flux differential caused by running a d-axis current into the first pole-pair while setting the d-axis currents in the other pole-pairs to zero was calculated; results are displayed in Fig. 4. The force caused by this flux imbalance can be calculated using Maxwell's stress tensor.

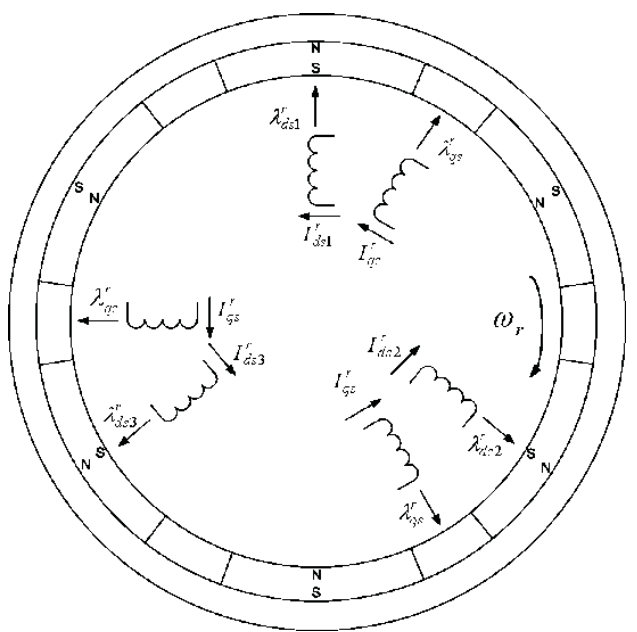

Fig. 3. Pole-Pairs in Rotor Reference Frame

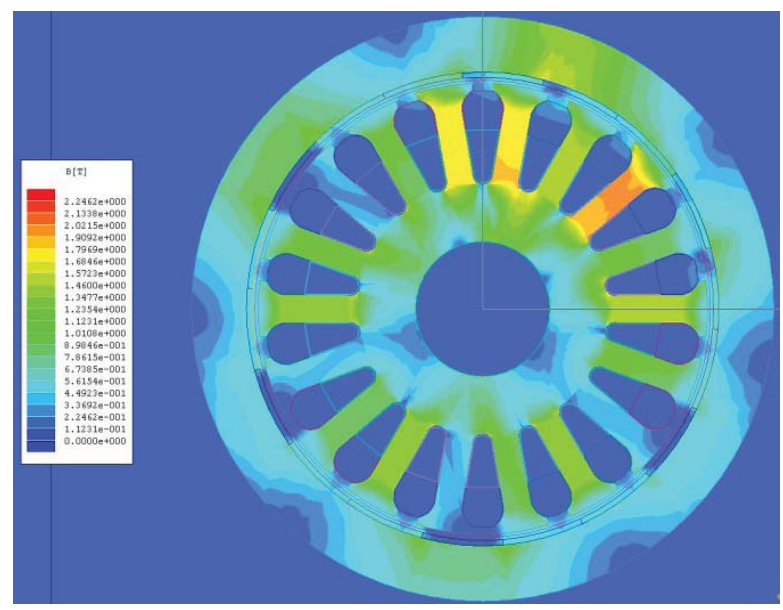

Fig. 4. FEM Flux Imbalance

The induced force caused by a flux imbalance is attractive and in line with the air gap; because of this, a radial motor can only produce radially directed forces, and an axial motor can only produce axially directed forces. The prototype motors examined by this paper employ a conical air-gap to create axial as well as radial force components. This machine uses two motors with opposing cones, as seen in Fig. 5. Fig. 6 shows the available force vectors in the two motor planes.

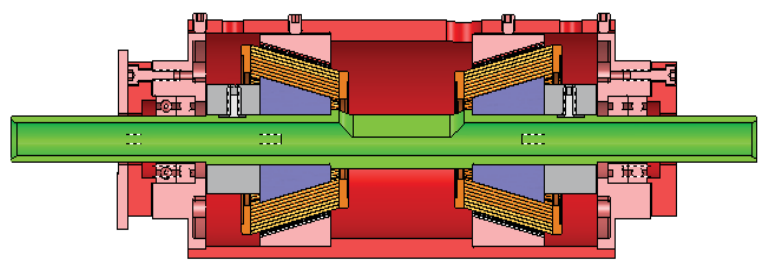

Fig. 5. Pair of Opposed Bearingless Conical Motors

A motor control system, with associated power electronics and sensors, was built to run the prototype machine. In this controller, five PID position control loops determine the force applied to the rotor while levitating; two for each radial plane, and one for the axial direction. While levitating and rotating, 
position measurements are taken with eddy current proximity sensors.

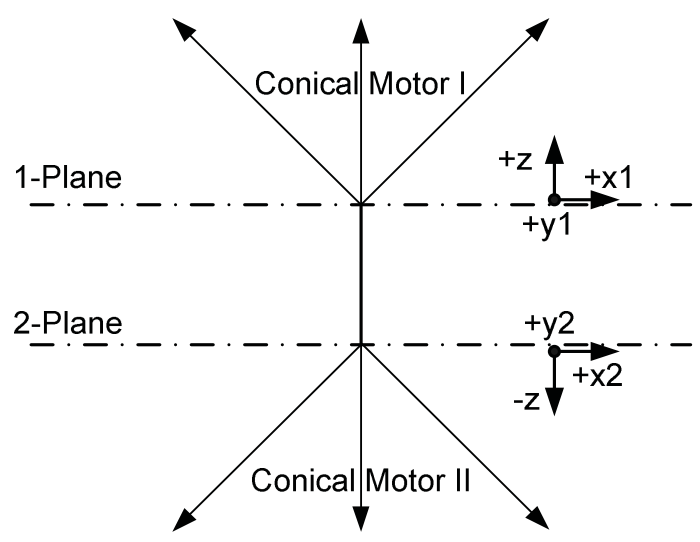

Fig. 6. Force Vectors from Both Motors

\section{SYNCHRONOUS WHIRL}

The main cause of synchronous whirl is rotor imbalance. Since this prototype machine was not designed go to high speed, the rotor was not mechanically balanced. Even if it had been balanced, some imbalance would still exist. Consider a disk with the center of mass offset from the geometric center [13], as seen in Fig. 7. The forces in the $\mathrm{x}$ and $\mathrm{y}$ direction are given by (1) and (2) respectively.

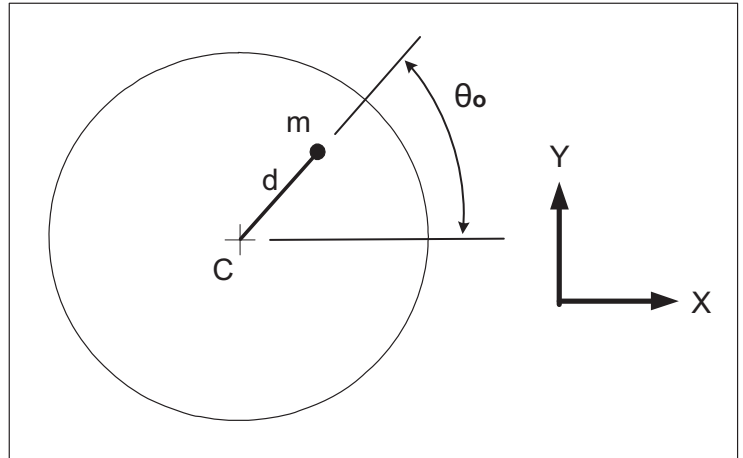

Fig. 7: Imbalanced Rotor

$$
\begin{gathered}
\mathrm{F}_{\mathrm{x} \text {-imbalance }}=\mathrm{m} \cdot \mathrm{d} \cdot \omega^{2} \cdot \cos \left(\omega \cdot \mathrm{t}+\theta_{\mathrm{o}}\right) \\
\mathrm{F}_{\mathrm{y} \text {-imbalance }}=\mathrm{m} \cdot \mathrm{d} \cdot \omega^{2} \cdot \sin \left(\omega \cdot \mathrm{t}+\theta_{\mathrm{o}}\right)
\end{gathered}
$$

Where $\omega$ is the angular velocity, $m$ is the mass, and $d$ is the distance the center of mass is offset from the geometric center of the imbalanced disk. The equations of motion can be expressed as [13]:

$$
\begin{aligned}
& \mathrm{m} \cdot \ddot{\mathrm{X}}+c \cdot \dot{X}+k \cdot X=\mathrm{F}_{\mathrm{x} \text {-imbalance }} \\
& =\mathrm{m} \cdot \mathrm{d} \cdot \omega^{2} \cdot \cos \left(\omega \cdot \mathrm{t}+\theta_{\mathrm{o}}\right) \\
& \mathrm{m} \cdot \ddot{\mathrm{Y}}+c \cdot \dot{Y}+k \cdot Y=\mathrm{F}_{\mathrm{y}-\text { imbalance }} \\
& =\mathrm{m} \cdot \mathrm{d} \cdot \omega^{2} \cdot \sin \left(\omega \cdot \mathrm{t}+\theta_{\mathrm{o}}\right)
\end{aligned}
$$

Where $k$ is the spring constant and $c$ is the damping coefficient of the disk's suspension; in this case, these are defined by the gains of the PID controller. These equations can be written more compactly in complex form; first the imbalance force can be expressed as:

$$
\begin{aligned}
& F_{\text {imbalance }}=F_{x-\text { imbalance }}+i \cdot F_{y \text {-imbalance }} \\
& =m \cdot d \cdot \omega^{2} \cdot\left(\begin{array}{l}
\cos \left(\omega \cdot t+\theta_{0}\right) \\
+i \cdot \sin \left(\omega \cdot t+\theta_{0}\right)
\end{array}\right)
\end{aligned}
$$

This can be simplified with Euler's formula:

$$
\begin{aligned}
& F_{\text {imbalance }}=m \cdot d \cdot \omega^{2} \cdot e^{i\left(\omega t+\theta_{0}\right)} \\
& =m \cdot d \cdot \omega^{2} \cdot e^{i \cdot \theta_{0}} \cdot e^{i \cdot \omega \cdot t}
\end{aligned}
$$

The equations of motion can be written as:

$$
m \cdot \ddot{P}_{x y}+c \cdot \dot{P}_{x y}+k \cdot P_{x y}=F_{\text {imbalance }}
$$

Where $P_{x y}$ is the position expressed as a complex number. The Laplace transformation of the imbalance force is:

$$
\mathscr{L}\left(F_{\text {imbalance }}\right)=m \cdot d \cdot \omega^{2} \cdot e^{i \cdot \theta_{0}} \cdot \frac{1}{s-i \cdot \omega}
$$

Now by substituting (8) into the Laplace transformation of (7):

$$
\begin{aligned}
& m \cdot s^{2} \cdot P_{x y}+c \cdot s \cdot P_{x y}+k \cdot P_{x y} \\
& =m \cdot d \cdot \omega^{2} \cdot e^{i \cdot \theta_{0}} \cdot \frac{1}{s-i \cdot \omega}
\end{aligned}
$$

The position can now be expressed as:

$$
\begin{aligned}
& P_{x y}=\frac{m \cdot d \cdot \omega^{2} \cdot e^{i \cdot \theta_{0}}}{\left(m \cdot s^{2}+c \cdot s+k\right)(s-i \cdot \omega)} \\
& =\frac{d \cdot \omega^{2} \cdot e^{i \cdot \theta_{0}}}{\left(s^{2}+\frac{c}{m} \cdot s+\frac{k}{m}\right)(s-i \cdot \omega)}
\end{aligned}
$$

This can be simplified with partial fraction expansion:

$$
\begin{aligned}
& P_{x y}=\frac{A}{s+\frac{c-\sqrt{c^{2}-4 \cdot m \cdot k}}{2 \cdot m}} \\
& +\frac{B}{s+\frac{c+\sqrt{c^{2}-4 \cdot m \cdot k}}{2 \cdot m}}+\frac{C}{(s-i \cdot \omega)}
\end{aligned}
$$

Equation (11) does not have RHP poles, because the system is assumed to be stable:

$$
\operatorname{Re} a l\left(\frac{c-\sqrt{c^{2}-4 \cdot m \cdot k}}{2 \cdot m}\right)>0
$$

and

$$
\operatorname{Re} a l\left(\frac{c+\sqrt{c^{2}-4 \cdot m \cdot k}}{2 \cdot m}\right)>0
$$

The coefficients are:

$$
\begin{gathered}
\left.A=\frac{\frac{2 \cdot m^{2} \cdot d \cdot \omega^{2} \cdot e^{i \cdot \theta_{0}}}{\sqrt{c^{2}-4 \cdot m \cdot k}}}{\left(-2 \cdot i \cdot \omega \cdot m-c+\sqrt{c^{2}-4 \cdot m \cdot k}\right.}\right) \\
\frac{2 \cdot m^{2} \cdot d \cdot \omega^{2} \cdot e^{i \cdot \theta_{0}}}{\sqrt{c^{2}-4 \cdot m \cdot k}} \\
\left.B=\frac{\sqrt{c^{2}-4 \cdot m \cdot k}}{2 \cdot i \cdot \omega \cdot m+c+\sqrt{2}}\right)
\end{gathered}
$$




$$
C=\frac{\left(-2 \cdot i \cdot \omega \cdot m-c+\sqrt{c^{2}-4 \cdot m \cdot k}\right)}{\left(2 \cdot i \cdot \omega \cdot m+c+\sqrt{c^{2}-4 \cdot m \cdot k}\right)}
$$

Now the inverse Laplace transform is taken on the position:

$$
\begin{aligned}
& P_{x y}= \\
& A \cdot e^{-\frac{c-\sqrt{c^{2}-4 \cdot m \cdot k}}{2 \cdot m} \cdot t}+B \cdot e^{-\frac{c+\sqrt{c^{2}-4 \cdot m \cdot k}}{2 \cdot m} \cdot t}+C \cdot e^{i \cdot \cdot \omega t}
\end{aligned}
$$

For a stable system, the first two terms approach zero as $\mathrm{t}$ approaches infinity. The steady state solution is the third term. The magnitude of $\mathrm{C}$ can be expressed as:

$$
|C|=\frac{\omega^{2} \cdot d}{\sqrt{\left(\frac{k}{m}-\omega^{2}\right)^{2}+\left(\frac{c \cdot \omega}{m}\right)^{2}}}
$$

The phase of $\mathrm{C}$ can be written as:

$$
\angle C=\tan ^{-1}\left(\frac{c \cdot \omega}{m\left(\frac{k}{m}-\omega^{2}\right)}\right)+\theta_{0}=\beta+\theta_{0}
$$

So the whirl (position of rotor center of geometry) leads the imbalance (position of the center of mass) by an angle of $\beta$. The magnitude of the whirl hits a maximum when the angular velocity is:

$$
\omega_{\text {critical }}=\sqrt{\frac{k}{m}}
$$

This speed is known as a critical speed. The magnitude of the whirl at this critical speed is:

$$
|C|_{\omega=\omega_{\text {critical }}}=\frac{d \cdot \sqrt{k \cdot m}}{c}
$$

It is seen that damping is required to limit the magnitude of this whirl at the critical speed. Equation (19) shows that the angle of the whirl of the center of geometry $(\beta)$ with respect to the angle of the center of mass $\left(\theta_{0}\right)$ starts at zero degrees, then it increases to 90 degrees as the rotor approaches the critical speed. After the rotor surpasses the critical speed the the angle $(\beta)$ jumps to -90 degrees; as speed continues to increase the angle $(\beta)$ asymptotically approaches 0 degrees. It is seen from (18) that as speed is increased from the critical speed, the magnitude of the imbalance approaches the distance between the center of mass and center of geometry. This means that the center of geometry of the rotor is rotating around the rotor's center of mass.

\section{FEEDFORWARD COMPENSATION}

Typically imbalance is mitigated with feed-forward for subcritical speeds. This can be thought of as electrically balancing the rotor. When the rotor is at supercritical speeds ( $\omega>\omega_{\text {critical }}$ ), synchronous notch filters are used on the position feedback, because the rotor spins around its center of mass. At subcritical speeds $\left(\omega<\omega_{\text {critical }}\right)$ this will not work, because feedback is necessary to counteract the imbalance force. In this case rotor imbalance can be counteracted with a feedforward signal.

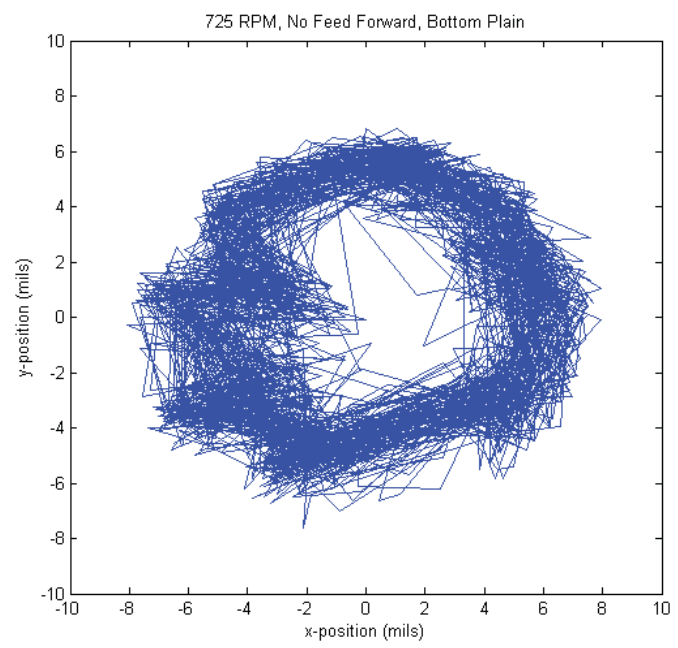

Fig. 8: $\mathrm{x}$ and y position, bottom plain, no feedforward

Fig. 8 shows the $\mathrm{x}$ and $\mathrm{y}$ position of the rotor in the bottom sensor plane. The measured position is the center of geometry of the rotor; note the circular whirl. Now considering the rotor position time domain signal as complex, with the $\mathrm{x}$ directed component considered real and the $\mathrm{y}$ directed component considered imaginary, the FFT was taken and is shown in Fig. 9.

The synchronous components are at $\pm 12 \mathrm{~Hz}$. Notice that there are different components for positive and negative frequencies. To consider the meaning of this note the synchronous component can be expressed as:

$$
\begin{aligned}
& F(w)=\mathcal{Z}(f(t)) \\
& =\delta\left(\omega_{s y n c}\right) \cdot A_{f-\text { sync }} \cdot e^{i \cdot \theta_{f-s y n c}} \\
& +\delta\left(-\omega_{s y n c}\right) \cdot A_{b-s y n c} \cdot e^{i \cdot \theta_{b-s y n c}}
\end{aligned}
$$

Where $\omega_{\text {sync }}$ is the angular velocity of the rotor, $A_{f-s y n c}$ and $A_{b-s y n c}$ and $\theta_{f-s y n c}$ and $\theta_{b-s y c}$ are the magnitudes and angles of the positive and negative synchronous rotor displacements. 


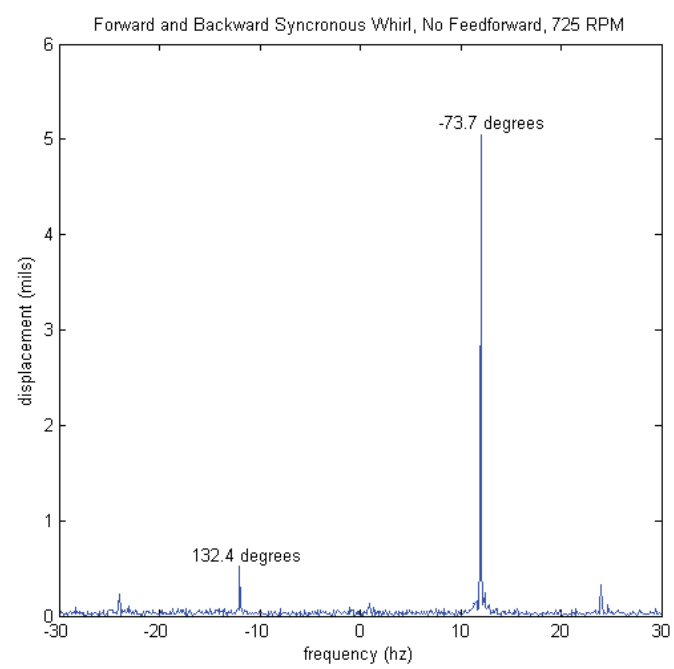

Fig. 9: FFT bottom plain, no feedforward

The inverse Fourier transform is expressed as:

$$
f(t)=\frac{1}{2 \pi} \int_{-\infty}^{\infty} F(\omega) \cdot e^{i \cdot \omega \cdot t} \cdot d \omega
$$

Which can be applied to (22):

$$
\begin{aligned}
& f(t)=\frac{A_{f-s y n c}}{2 \pi} \cdot e^{i \cdot \theta_{f-s y n c}} \cdot e^{i \cdot \omega_{s y n c} \cdot t} \\
& -\frac{A_{b-s y n c}}{2 \pi} \cdot e^{i \cdot \theta_{b-s y n c}} \cdot e^{-i \cdot \omega_{s y n c} \cdot t}
\end{aligned}
$$

Where

$$
\begin{aligned}
& e^{i \cdot \theta_{f-s y n c}} \cdot e^{i \cdot \omega_{s y n c} \cdot t} \\
& =\cos \left(\omega_{s y n c} \cdot t+\theta_{f-s y n c}\right) \\
& +i \cdot \sin \left(\omega_{\text {sync }} \cdot t+\theta_{f-s y n c}\right)
\end{aligned}
$$

and

$$
\begin{aligned}
& e^{i \cdot \theta_{b-s y n c}} \cdot e^{-i \cdot \omega_{s y n c} \cdot t} \\
& =\cos \left(-\omega_{\text {sync }} \cdot t+\theta_{b-s y n c}\right) \\
& +i \cdot \sin \left(-\omega_{s y n c} \cdot t+\theta_{b-s y n c}\right)
\end{aligned}
$$

This implies a circular component in the forward direction and a circular component in the reverse direction. The interference of these forward and backward components will create an elliptical shape in the measured position orbit.

Given this, a feed-forward signal is added to the measured $\mathrm{x}$ and $\mathrm{y}$ positions, in general this signal would have a magnitude and phase in both the positive and negative directions:

$$
\text { Pos }=x_{\text {meas }}+i \cdot y_{\text {meas }}+f f\left(\theta_{\text {mechanical }}\right)
$$

Now (24) is rewritten in terms of mechanical angle.

$$
\begin{aligned}
& f f\left(\theta_{\text {mechanical }}\right)= \\
& \frac{A_{f-\text { sync }}}{2 \pi} \cdot e^{i \cdot\left(\theta_{\text {mechanical }}+\theta_{f-\text { sync }}\right)} \\
& -\frac{A_{b-\text { sync }}}{2 \pi} \cdot e^{-i \cdot\left(\theta_{\text {mechanical }}+\theta_{b-\text { sync }}\right)}
\end{aligned}
$$

Since the imbalance excitation changes as speed increases, this feed-forward signal must change as speed increases. In practice the feed-forward signal is tuned at specific rotor speeds and the gains and magnitudes are interpolated between these speed points. One method of tuning would be to take the FFT of rotor position without feed-forward compensation, then the compensating feed-forward signal would have the same forward and backward amplitudes, but the phases would be shifted by 180 degrees. This feed-forward signal would be added to the position commands. The only disadvantage of this method is that it requires that feed-forward be off for the measurement, and for extremely unbalanced rotors, this might not be possible without touching the touchdown bearings. But this same process could be employed in stages where some sub-optimal amount of feed-forward is used. Then the new feed-forward signal is the sum of the old feed-forward signal and the one calculated from the FFT of rotor position.

In implementation the feed-forward was tuned every one hundred RPM, up to 1000 RPM. (Note that the motor was designed for a high speed flywheel application of 100000 RPM. However, due to the complex shape of the stator and limitations in the available budget, a non-laminated stator design had to be used, and thus the prototype tested in this paper can only be run at lower speeds). For simplicity only the forward rotating feed-forward components were used,

because they were much larger than the backward components. The tuning strategy was to simply adjust the gain and phase of the feed-forward signal until the circular shape of the position was minimized.

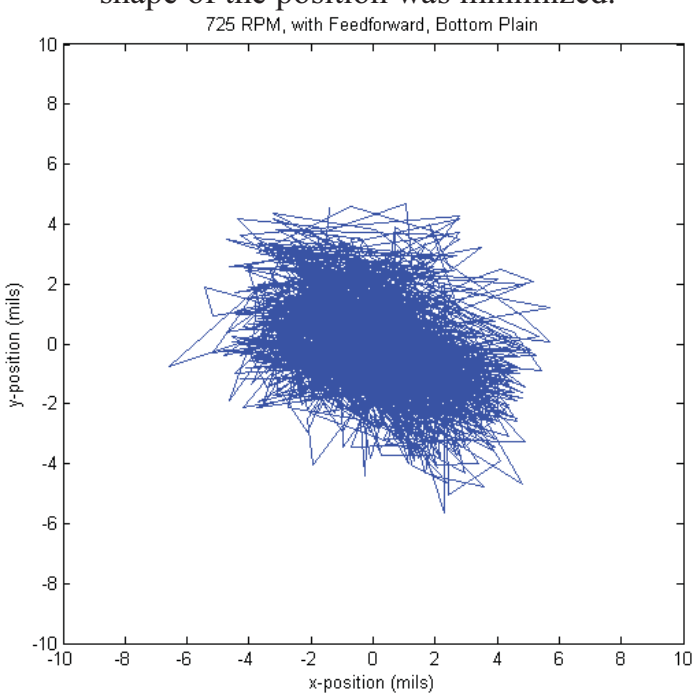

Fig. 10: $\mathrm{x}$ and $\mathrm{y}$ position with feed-forward

The positions for the case of the rotor spinning at 725 RPM with the feed-forward command implemented are shown in Fig. 10. The FFT of this data is shown in Fig. 11. Note that the forward synchronous component has been reduced by about 4.5 times. The backward synchronous component is the same, as expected, because a backward component was not included in the feed-forward compensation. 


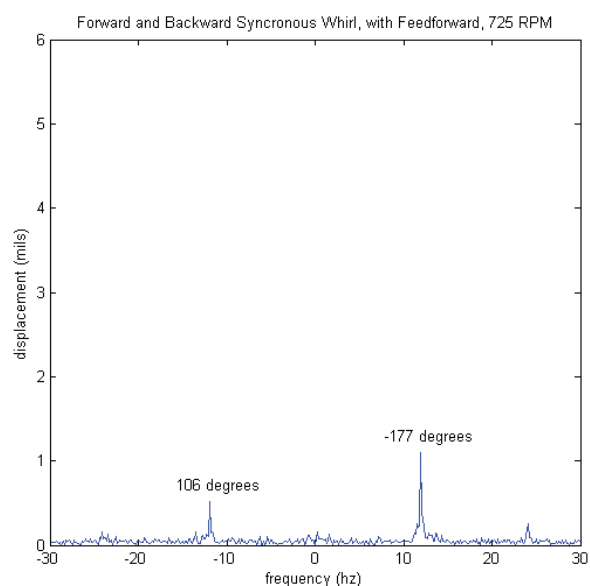

Fig. 11: FFT of bottom plane with feed-forward

\section{CONCLUSION}

This paper examined the effect of imbalance on a bearingless motor system. It was shown that feedforward can be used to reduce the synchronous whirl of the rotor due to imbalance.

\section{REFERENCES}

[1] A. Chiba, K. Chida and T. Fukao, "Principles and Characteristics of a Reluctance Motor with Windings of Magnetic Bearing", in Proceedings of International Power Electronic Conference (IPEC-Tokyo), 1990, pp. 919-926.

[2] A. Chiba, M. A. Rahman and T. Fukao, "Radial Force in a Bearingless Reluctance Motor", IEEE Transactions on Magnetics, Volume 27, Issue 2, pp. 786-790, March 1991.

[3] A. Chiba, D. T. Power, M. A. Rahman, "No Load Characteristics of a Bearingless Induction Motor", IEEE Transactions on Magnetics, Volume 27, Issue 6, pp. 5199-5201, November 1991.

[4] K. Dejima, T. Ohishi, and Y. Okada, "Analysis and control of a permanent magnet type levitated rotating motor," in IEEJ Proceedings of Symposium Dynamics of Electro Magnetic Force, June 1992, pp. 251256.

[5] K. Shimada, M. Takemoto, A. Chiba, and T. Fukao, "A Stable Rotation in Switched Reluctance Type Bearingless Motors", in Papers of IEEJ Technical Meeting on Linear Drive, 1997, LD-97-116.

[6] P. Kascak, "Fully Levitated Rotor Magnetically Suspended by Two Pole-Pair Separated Conical Motors," Ph.D. Thesis, Case Western Reserve University, Cleveland, OH, 2010.

[7] P. Kascak, R. Jansen, and T. Dever, "Control System for Bearingless Motor-Generator", U.S. Patent 7,456,537 B1, Nov. 25, 2008.

[8] P. Kascak, R. Jansen, and T. Dever, "Control System for Bearingless Motor-Generator", U.S. Patent 7,667,418, Feb. 23, 2010.

[9] P. Kascak, R. Jansen, T. Dever, A. Nagorny, and K. Loparo "Bearingless Five-Axis Rotor Levitation with Two Pole Pair Separated Conical Motors", in the Conference Record of IEEE Industrial Applications Society Annual Meeting, October 2009.

[10] P. Kascak, T. Dever, R. Jansen, "Conical bearingless motor-generators", in McGraw-Hill Yearbook of Science \& Technology, 2010 edition, pp. 81-84.

[11] P. Kascak, T. Dever, R. Jansen, A. Nagorny, and K. Loparo, "Motoring Performance of a Conical Pole-Pair Separated Bearingless Electric Machine", in the Conference Record of IEEE EnergyTech, May 2011.

[12] P. Kascak, R. Jansen, T. Dever, A. Nagorny, K. Loparo, "Levitation Performance Of Two Opposed Permanent Magnet Pole-Pair Separated Conical Bearingless Motors", in the Conference Record of the IEEE Energy Conversion Congress and Exposition, Phoenix, AZ, September 2011.

[13] Vance, J. M., Rotordynamics of Turbomachinery, John Wiley \& Sons, Inc., New York, New York, 1988
[14] T. Dever, G. Brown, R. Jansen, P. Kascak, A. Provenza, "Magnetic Bearing Controller Improvements for High Speed Flywheel Systems", IECEC, Portsmouth, Virginia, August 2003.

\section{BIOGRAPHIES}

Peter Kascak received his Bachelor of Science degree in Electrical and Computer Engineering in 1997 and his Master's degree in Electrical Engineering in 1999 from The Ohio State University. In 2010, Dr. Kascak received the Ph.D. in Electrical Engineering degree from Case Western Reserve University with the thesis titled "Fully Levitated Rotor Magnetically Suspended by Two Pole-Pair Separated Conical Motors".

Ralph Jansen received the Bachelor of Fluid and Thermal Engineering degree and the Master of Mechanical Engineering degree from Case Western Reserve University in 1991 and 1995 respectively.

Timothy Dever received his Bachelor's degree in Electrical Engineering from Cleveland State University in 1987. In 1991, Mr. Dever received his Master's degree in Electrical Engineering from Case Western Reserve University.

Aleksandr Nagorny received his Bachelor degree in Electric Machines in 1978 from Odessa National Polytechnic University (Ukraine) and his Ph.D. degree in Electrical Engineering (Electric Machines) in 1990 from Kharkov State Polytechnic University (Ukraine). He also received his Master of Science degree in Electrical Engineering in 2003 from Oregon State University. Currently he is working in Resmed Motor Technologies Inc. designing high speed motors for medical applications.

Kenneth Loparo received the Ph.D. degree in Systems and Control Engineering from Case Western Reserve University, Cleveland, Ohio, in 1977. He was an Assistant Professor in the Mechanical Engineering Department at Cleveland State University from 1977 to 1979 and he has been on the faculty of The Case School of Engineering, Case Western Reserve University since 1979. 